\title{
Editorial
}

\section{Epidemiología del aborto y su prevención en Chile}

Independiente de su estatus legal, la prevención del aborto provocado continúa siendo un objetivo clave en la mayor parte del mundo occidental $[1,2]$. Chile no es la excepción a la regla. De hecho, nuestro país cuenta con una interesante serie de datos epidemiológicos e investigaciones recientes en la materia -algunas conducidas por nuestro Instituto- que intentaré sintetizar brevemente en este artículo editorial.

La mortalidad por aborto ha experimentado una reducción continua en los últimos 50 años [3], al punto que la muerte por aborto provocado ha llegado a ser un fenómeno excepcional en términos epidemiológicos (riesgo de 1 en 4 millones de mujeres en edad fértil o 0,4 por 100.000 nacidos vivos para cualquier tipo de aborto excluyendo embarazo ectópico). Luego del cambio de ley en 1989 [4], las muertes por aborto continuaron disminuyendo, desde 10,8 hasta 0,39 por 100.000 nacidos vivos (Figura 1), lo cual, como materia de hecho científico, desafía la noción que una legislación menos permisiva conduce a mayor mortalidad por aborto [3,5-7].

Varios factores habrían contribuido al fenómeno observado en Chile. La reducción de la fecundidad (de 5 a 1,8 hijos por mujer en 50 años), con el acceso creciente a métodos de planificación familiar desde 1964 [8], el incremento de la escolaridad femenina (de 3,5 a 12 años promedio en 50 años), expansión progresiva de la atención obstétrica de emergencia, acceso precoz al control del embarazo y cuidados post-aborto, aparecen como los más relevantes en estudios recientes $[9,10]$. Es de notar que el incremento de la educación mostró efectos sinérgicos, modulando el impacto de las otras variables [10]. Así mismo, la evidencia sugiere que una lex artis dinámica, junto con una ética médica reflexiva aplicada caso a caso [11], han prevalecido en la medicina obstétrica chilena, haciendo innecesario volver a una legislación particular para casos extremos de compromiso vital materno. Incluso, una legislación tal, podría ser regresiva dependiendo de su alcance, interpretación y uso, como históricamente ocurrió con la ley anterior $[12,13]$. Es interesante un estudio de serie temporal con 100 años de registros oficiales [3], constatando que la razón de mortalidad materna global, aumentó durante los primeros siete años de vigencia de la primera ley de aborto terapéutico promulgada en 1931, alcanzando un pico histórico de 989,2 muertes por 100.000 nacidos vivos en 1937, la más alta en la historia de la salud materna chilena del siglo XX (Figura 2).

La morbilidad por aborto también ha disminuido en las estadísticas oficiales [9]. Por ejemplo, en 1965 los egresos hospitalarios por cualquier tipo de aborto (espontáneo o provocado) fueron 56.130 , es decir, $18,6 \%$ sobre el total de nacidos vivos ese año, ocupando casi un tercio de las camas obstétricas disponibles en aquella época [14]. En la actualidad, los egresos por aborto rondan los 30.000 por año, i.e. $12 \%$ sobre el total de nacidos vivos, ocupando $10 \%$ a $15 \%$ de las camas obstétricas disponibles. En la última década (2001-2011), no obstante las hospitalizaciones obstétricas incluyendo partos, permanece constante (33\% del total de egresos en mujeres), se observa una importante disminución en las tasas de hospitalización por aborto (Figura 3).

Al desagregar los nueve códigos diagnósticos del grupo $O$ por causas o desenlaces abortivos de la décima Clasificación Internacional de Enfermedades (CIE-10), se observa que el embarazo ectópico (Cod. O00), embarazo molar, otros productos anormales de la concepción (Cod. O01, 002 y O08), y el aborto espontáneo (Cod. O03), han permanecido con tasas remarcablemente constantes, representado $70 \%$ del total de egresos por aborto en el último año de la serie. Como expone una revisión reciente sobre el uso de los códigos de la CIE-10 para desenlaces abortivos [15], ninguno de los anteriores se relaciona con el aborto provocado en la clandestinidad. Por ejemplo, el código $\mathrm{O} 02$ se utiliza, entre otros, para clasificar el embarazo anembrionado [16] terminado con legrado, siendo frecuente en Chile debido a la alta medicalización obstétrica ecográfica y quirúrgica, especialmente en el sector privado. Es un error común $[17,18]$, considerar el código $\mathrm{O} 02$ como un estimador de abortos ilegales o clandestinos, lo cual abultará injustificadamente los números. 


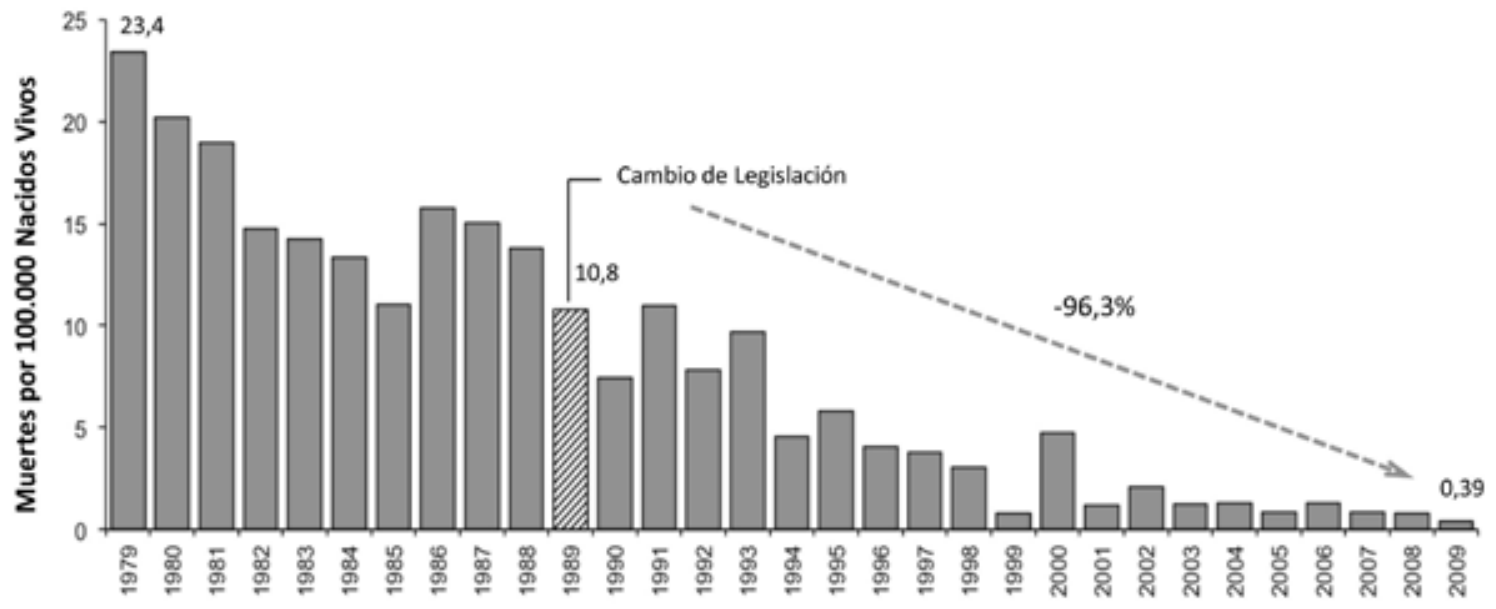

Figura 1. Razón de mortalidad materna por aborto (muertes por cada 100.000 nacidos vivos excluido el embarazo ectópico) entre 1979 y 2009 en Chile [3]. Luego de 1989 (derogación de código sanitario que autorizaba el aborto terapéutico con la firma de dos médicos) la mortalidad continuó disminuyendo (96\% en 20 años).

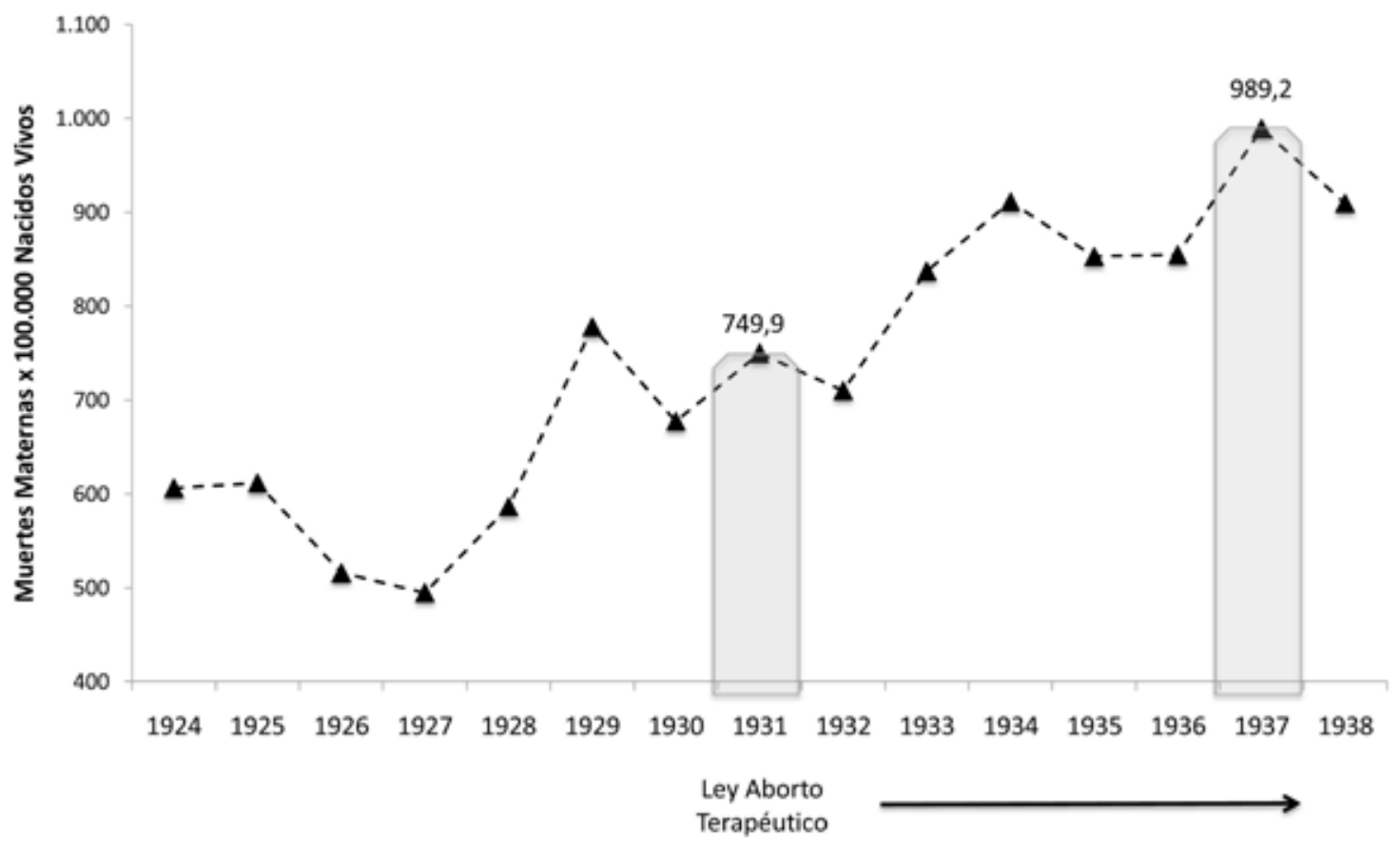

Figura 2. Razón de mortalidad materna en Chile, 1924 - 1938 [3]. 


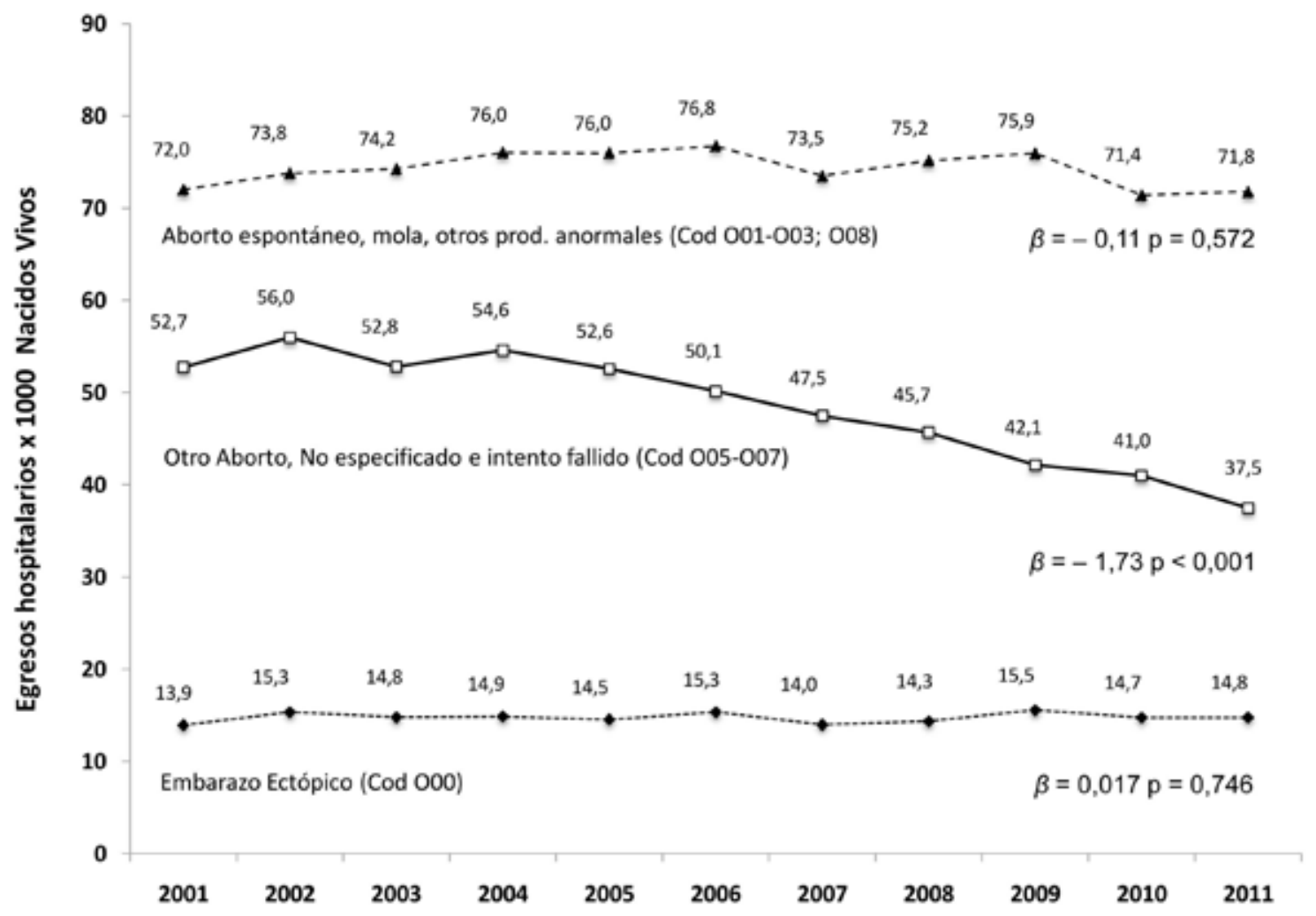

Figura 3. Morbilidad por aborto en Chile según estadísticas oficiales de egresos hospitalarios por diferentes tipos de aborto, 2001 - 2011. En los códigos de egreso O05, O06 y O07 (línea sólida), se sospechan complicaciones por abortos provocados. La tendencia anual fue calculada a través de coeficientes $\beta$ estimados por regresión simple. Fuente: Datos oficiales del Ministerio de Salud, Departamento de Estadísticas en Salud (DEIS).

En contraste, la Figura 3 muestra que los egresos por otro aborto (O05), aborto no especificado (O06) e intento fallido de aborto (O07) agrupados, presentan una tendencia a la baja de $2 \%$ por año o 1,73 por 1000 nacidos vivos por año $(r=0,94$; $\beta=1,74 ; p<0,001)$. Es en estos códigos de egreso, particularmente O06, en los cuales se sospecha la presencia de complicaciones por abortos provocados ilegítimos [15]. Sin embargo, no todos los abortos clasificados como aborto no especificado, corresponderán necesariamente a complicaciones secundarias al aborto inducido en la clandestinidad, pues se utiliza indistintamente para contar estadísticamente abortos en los que el médico no ha consignado otro diagnóstico o causa específica en el registro clínico (e.g. "aborto, legrado" o simplemente "aborto" o "legrado" son registros clínicos comunes que se clasifican como 006), y en los que no se realiza estudio patológico. De esta manera, permanece aún altamente especulativo, asumir que todos los egresos con desenlace abortivo clasificados en el código O06 correspondan a complicaciones de aborto provocado, como propone Molina et al., en un trabajo reciente [17].

Es de notar que el código 006 (aborto no especificado) concentra $28,8 \%$ del total de egresos por embarazos con desenlace abortivo (8.892 de un total de 30.860 para el último año estadístico disponible) y la reducción de egresos en este sólo código, explica prácticamente la totalidad de la reducción de la morbilidad por aborto en la última década, con una caída global sobre $15 \%$. Dado que la tasa por otros desenlaces abortivos de causa conocida ha permanecido constante en el tiempo (Figura 3), la reducción no parece ser un artefacto de clasificación (i.e. intercambio de códigos). Tampoco hemos detectado en las estadísticas oficiales, un reemplazo significativo de hospitalizaciones por apendicitis u otras cirugías comunes, parte de un mito urbano bastante extendido en la opinión pública nacional. 
Se trata al parecer, de una reducción de morbilidad por aborto real, consistente y significativa en términos epidemiológicos.

En términos de su plausibilidad, varios factores podrían explicar una disminución de la magnitud del aborto provocado y de sus complicaciones en la última década. Por ejemplo, el uso de fármacos con efecto abortivo como misoprostol, cada vez más accesible en el mercado informal $[19,20]$, ha sido un factor mencionado en la literatura $[5,9,17,20]$. Sin embargo, es necesario señalar que más de la mitad de las mujeres que lo utilizan pueden percibir sangrado y dolor pélvico mayor al de un ciclo menstrual regular, buscando asistencia médica. Además, la tasa de complicaciones y fallas puede alcanzar $30 \%$ o más con el misoprostol auto-administrado, utilizado en dosis inadecuadas o sobre las 9 semanas de gestación [21-23]. Así, cabría esperar que cualquier incremento importante en magnitud del uso ilegítimo de misoprostol a nivel de población, se traslade también a un incremento de la morbilidad hospitalaria por aborto, particularmente en el código 006 (aborto no especificado). Como se ha observado lo contrario, es difícil que un incremento en el uso de este fármaco explique por sí solo la tendencia observada en la Figura 3.

Un segundo factor que podría explicar la reducción de morbilidad por aborto provocado, podría estar asociado con una mayor prevalencia o consistencia en el uso de métodos anticonceptivos, incluyendo el acceso creciente a contracepción de emergencia en la red de salud. En un estudio reciente de mujeres chilenas con embarazos no planificados vulnerables en riesgo de aborto [24], el $49,6 \%$ no estaba utilizando algún método de prevención al momento de la concepción, mientras $23,1 \%$ manifestó estar usando un método hormonal, $3,1 \%$ algún tipo de dispositivo intrauterino y $8,1 \%$ condón, sin diferencias entre mujeres que declaran o no declaran intención de aborto (Tabla I).

Tabla I

ALGUNAS CARACTERÍSTICAS BASALES SELECCIONADAS DE UN ESTUDIO EN CURSO EN UNA COHORTE [24,28] DE MUJERES CHILENAS CON EMBARAZOS NO PLANIFICADOS EN SITUACIÓN DE VULNERABILIDAD, DE ACUERDO A SI DECLARAN O NO DECLARAN INTENCIÓN DE ABORTO

\begin{tabular}{|c|c|c|c|c|}
\hline & \multirow{2}{*}{$\begin{array}{l}\text { Población total } \\
\qquad(\mathrm{n}=3134)\end{array}$} & \multicolumn{2}{|c|}{ Declara intención de aborto } & \multirow{2}{*}{ Valor $\mathrm{p}^{*}$} \\
\hline & & No $(n=2648)$ & $\mathrm{Si}(n=486)$ & \\
\hline Edad (media \pm ES) & $22,7 \pm 6.2$ & $22,7 \pm 6.2$ & $22,9 \pm 6.5$ & 0,363 \\
\hline \multicolumn{5}{|l|}{ Residencia (\%) } \\
\hline Región Metropolitana & 70,7 & 70,9 & 69,5 & \multirow{2}{*}{0,547} \\
\hline Otras regiones & 29,3 & 29,1 & 30,5 & \\
\hline \multicolumn{5}{|l|}{ Años de educación (\%) } \\
\hline 1 a 8 & 12,3 & 13,1 & 8,2 & 0,035 \\
\hline 9 a 12 & 56,7 & 57,3 & 53,7 & 0,121 \\
\hline 13 o más & 25,0 & 23,6 & 32,7 & $<0,001$ \\
\hline Indeterminado & 6,0 & 6,1 & 5,3 & 0,731 \\
\hline \multicolumn{5}{|l|}{ Trimestre de embarazo (\%) } \\
\hline Primero & 48,6 & 44,3 & 72,2 & $<0,001$ \\
\hline Segundo & 35,0 & 37,2 & 23,0 & $<0,001$ \\
\hline Tercero & 16,4 & 18,5 & 4,7 & $<0,001$ \\
\hline \multicolumn{5}{|l|}{ Método de prevención (\%) } \\
\hline Ninguno & 49,6 & 49,8 & 48,1 & 0,464 \\
\hline Anticonceptivo hormonal & 23,1 & 23,4 & 21,8 & 0,491 \\
\hline Dispositivo intrauterino (DIU) & 3,1 & 3,2 & 2,3 & 0,698 \\
\hline Condón & 8,1 & 7,7 & 10,5 & 0,228 \\
\hline Otro & 3,1 & 3,2 & 2,1 & 0,636 \\
\hline Indeterminado & 13,0 & 12,6 & 15,2 & 0,263 \\
\hline
\end{tabular}

*El valor $\mathrm{p}$ fue calculado mediante z-test para comparar 2 proporciones, excepto para medias de edad, comparadas mediante test de ANOVA. 
Aunque las tasas de abortos provocados en algunos países con leyes permisivas como España, aumentaron en paralelo con el incremento en la prevalencia global de métodos anticonceptivos [25], en Chile, está históricamente documentada una reducción de la morbilidad hospitalaria por aborto con la expansión de los programas de planificación familiar $[8,26]$. Una interesante hipótesis, es que el uso de métodos contraceptivos, podría ser más consistente o eficiente a través del tiempo con legislaciones de aborto menos permisivas. Por ejemplo, estudios recientes en series de tiempo en un panel de 41 países, utilizando reportes de gonorrea como proxy de conducta sexual riesgosa, muestran que el cambio hacia legislaciones más permisivas, se asoció a incrementos en la incidencia reportada de gonorrea, sugiriendo un aumento en la conducta sexual de riesgo (e.g. sexo sin protección) a nivel de poblaciones [27]. De acuerdo a los autores, la teoría económica predice que las leyes de aborto pueden afectar la conducta sexual, cambiando el costo marginal de tener relaciones sexuales riesgosas [27]. Mientras más permisivas, menor será dicho costo. Así, dependiendo de su permisividad, estas leyes podrían cambiar también el costo marginal de tener sexo riesgoso sin el uso de anticoncepción.

Un tercer factor a considerar en la disminución de morbilidad por aborto provocado, sería la emergencia y expansión gradual en nuestro país, de programas preventivos por parte de la sociedad civil (e.g. Fundación Chile Unido, Fundación San José, Proyecto Esperanza, Fundación Maternitas, ISFEM, etc.). Estos programas actúan identificando la situación de vulnerabilidad específica en embarazos no planificados en riesgo de aborto, brindando soporte y apoyo inmediato a la madre para resolverla. Recientemente, se presentaron en sesiones paralelas de discusión sobre las metas del milenio en Naciones Unidas [28], resultados preliminares de uno de los programas más extensos en cuanto a cobertura, con una cohorte de 3.134 mujeres chilenas cursando embarazos no planificados vulnerables. Como se observa en la Figura 4, según si la madre declara o no intención de aborto, el programa alcanzó un resultado de nacido vivo (con y sin adopción) que varía entre $69 \%$ y $85 \%$, dependiendo del grupo de riesgo y de si permanece o abandona el programa.

En el diseño de estrategias preventivas, la investigación para identificar situaciones de vulnerabilidad específica parece ser clave. En una sub-cohorte del estudio mencionado [28], con 486 mujeres que declararon una intención de aborto en la línea basal, seis factores explicaron más de 92\% de la motivación para abortar (Tabla II): coerción de padres o pareja o de un tercero con o sin violencia doméstica $(44,4 \%)$; interferencia con expectativas de vida (22,8\%); ocultamiento del embarazo por temor a reacción de padres o pareja $(20,4 \%)$; abuso sexual reiterado, violación e incesto $(2,1 \%)$; abandono de la pareja $(1,9 \%)$; y problemas de tipo psicológico o emocional $(1,9 \%)$. Sin aspirar a que esta muestra de alto riesgo -probablemente sesgada por autoselección- represente la realidad nacional, los resultados sugieren que la situación de vulnerabilidad específica para abortar, se relaciona con una problemática social que escapa al espectro de las acciones médicas habituales y que precisamente, los programas preventivos de la sociedad civil organizada parecen alcanzar más eficazmente.

Otra situación de embarazo vulnerable específica para el riesgo de aborto, lo constituyen las enfermedades congénitas $(3,1 \%$ de los nacidos vivos, alrededor de 7.400 nacimientos por año [29]), cuyo diagnóstico ocurre cada vez más temprano. Por ejemplo, en países europeos con leyes permisivas [30], el aborto provocado en casos de trisomía 21 (causa del síndrome de Down) alcanza entre 76\% y $96 \%$ de todos los casos diagnosticados. Considerando que estos países poseen en su mayor parte coberturas de pesquisa que superan $70 \%$ [30], se ha producido una reducción enorme de niños nacidos con esta condición [31]. Estudios sugieren que la implementación de programas de diagnóstico genético sistemáticos, incrementan las interrupciones del embarazo [32,33]. En la Figura 5, se compara la tasa de nacimientos con trisomía 21 en Chile [29] y países seleccionados [31,34]. Nuestro país, al igual que Irlanda, muestra una de las tasas más altas de nacimientos con síndrome de Down (2,47 por 1000 nacidos vivos, alrededor de 600 nuevos casos por año), sugiriendo que legislaciones menos permisivas, pueden contribuir a la prevención del aborto de estos niños en el tiempo [35]. Por otra parte, en el caso de enfermedades graves y letales como la anencefalia $(0,03 \%$ de nacidos vivos, alrededor de 65 a 75 casos por año) o la agenesia renal $(0,02 \%$, alrededor de 40 a 50 casos por año) el acceso a programas de cuidados paliativos perinatales por equipos obstétricos especializados, provee un solución alternativa eficaz al problema del aborto provocado [36-38]. Programas piloto de este tipo se han implementado en Santiago con éxito [39]. Es necesario expandir su cobertura a nivel nacional, tanto en el contexto de la salud materna y perinatal, como en la prevención del aborto provocado. 


\section{Tabla II}

\section{SITUACIONES DE VULNERABILIDAD ESPECÍFICA EN UN COHORTE DE 3.134 MUJERES CHILENAS} CON EMBARAZOS NO PLANIFICADOS EN RIESGO DE ABORTO [28]

\begin{tabular}{lcccc}
\hline & Población total & \multicolumn{2}{c}{ Declara intención de aborto } & Valor p† \\
& $(\mathrm{n}=3134)$ & No & Si \\
& $(\mathrm{n}=2648)$ & $(\mathrm{n}=486)$ & \\
\hline Coerción $^{\mathrm{a}}(\%)$ & 10,1 & 3,7 & 44,4 & $<0,001$ \\
Abuso sexual $^{\mathrm{b}}(\%)$ & 1,9 & 1,8 & 2,1 & 0,897 \\
Ocultar embarazo por temor $^{\mathrm{C}}(\%)$ & 14,1 & 12,9 & 20,4 & 0,001 \\
Expectativas de vida $^{\mathrm{d}}(\%)$ & 5,5 & 2,3 & 22,8 & $<0,001$ \\
Problemas psicológicos $^{\mathrm{e}}(\%)$ & 31,4 & 36,9 & 1,9 & $<0,001$ \\
Abandono de la pareja (\%) $_{\text {Abandono o expulsión del hogar (\%) }}$ & 7,0 & 7,9 & 1,9 & 0,009 \\
Asistencia para dar en adopción (\%) & 17,0 & 20,1 & 0,4 & $<0,001$ \\
Dudas sobre embarazo (\%) & 8,0 & 9,4 & - & - \\
Otras causas (\%) & 1,2 & 1,4 & - & - \\
\hline
\end{tabular}

${ }^{a}$ Coerción para abortar por parte de padre, madre, ambos padres o pareja; ${ }^{b}$ Violación, incesto o abuso sexual reiterado; ${ }^{C}$ Ocultar embarazo por temor a reacción de padres o pareja; ${ }^{d}$ Incluye no interrumpir estudios, temor a ser madre soltera, razones socioeconómicas y proyecto de vida; ${ }^{\mathrm{e}}$ Incluye abuso de drogas o alcohol; † z-test.

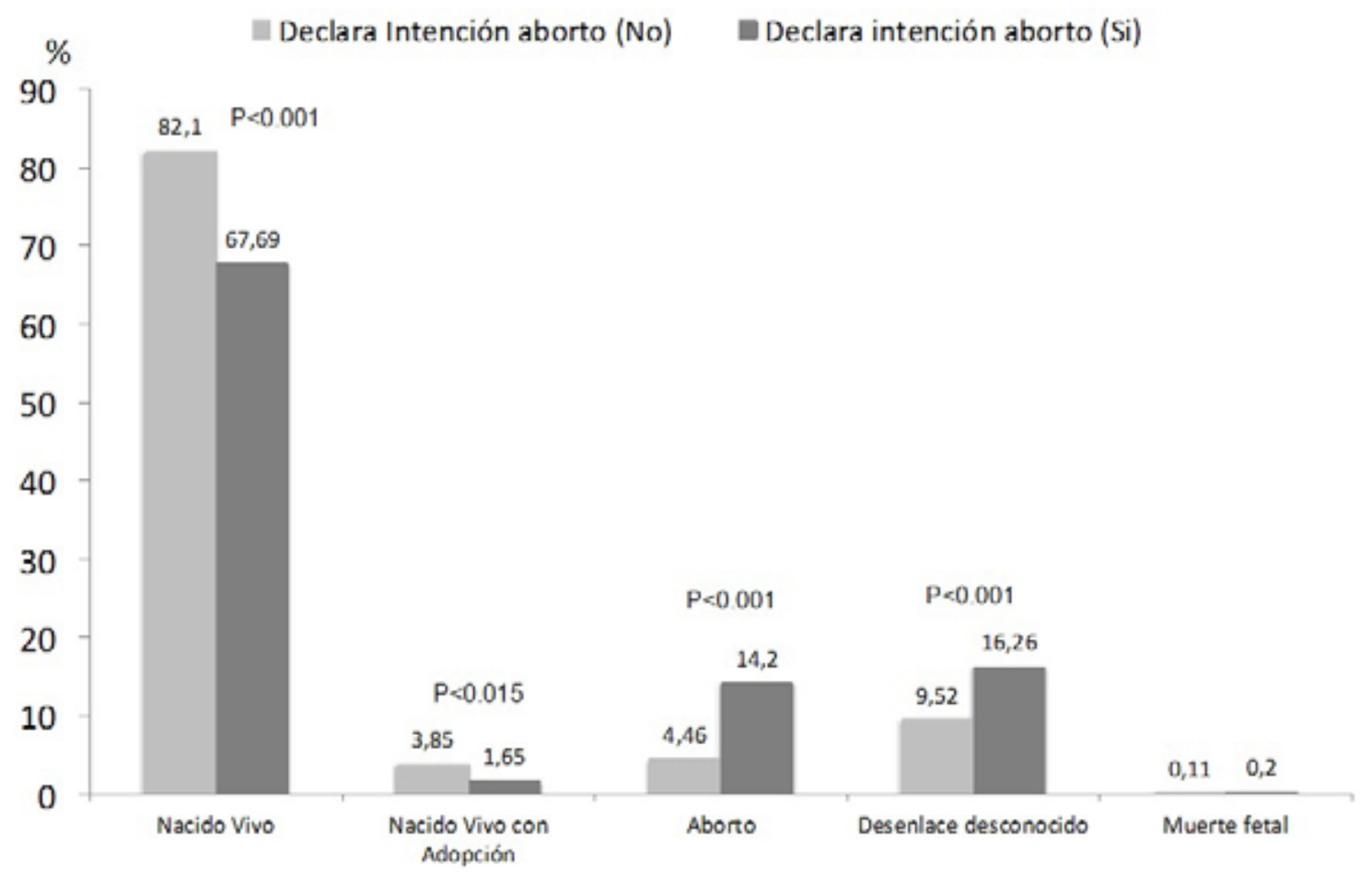

Figura 4. Desenlaces de un programa de soporte y apoyo de la sociedad civil (Fundación Chile Unido) en una cohorte de 3.134 mujeres chilenas con embarazos no planificados en situación de vulnerabilidad, agrupadas según si declaran intención de aborto $(\mathrm{No}=2.648 ; \mathrm{Si}=486)$. El desenlace desconocido corresponde a mujeres en las que se perdió contacto antes de las 20 semanas de gestación. 


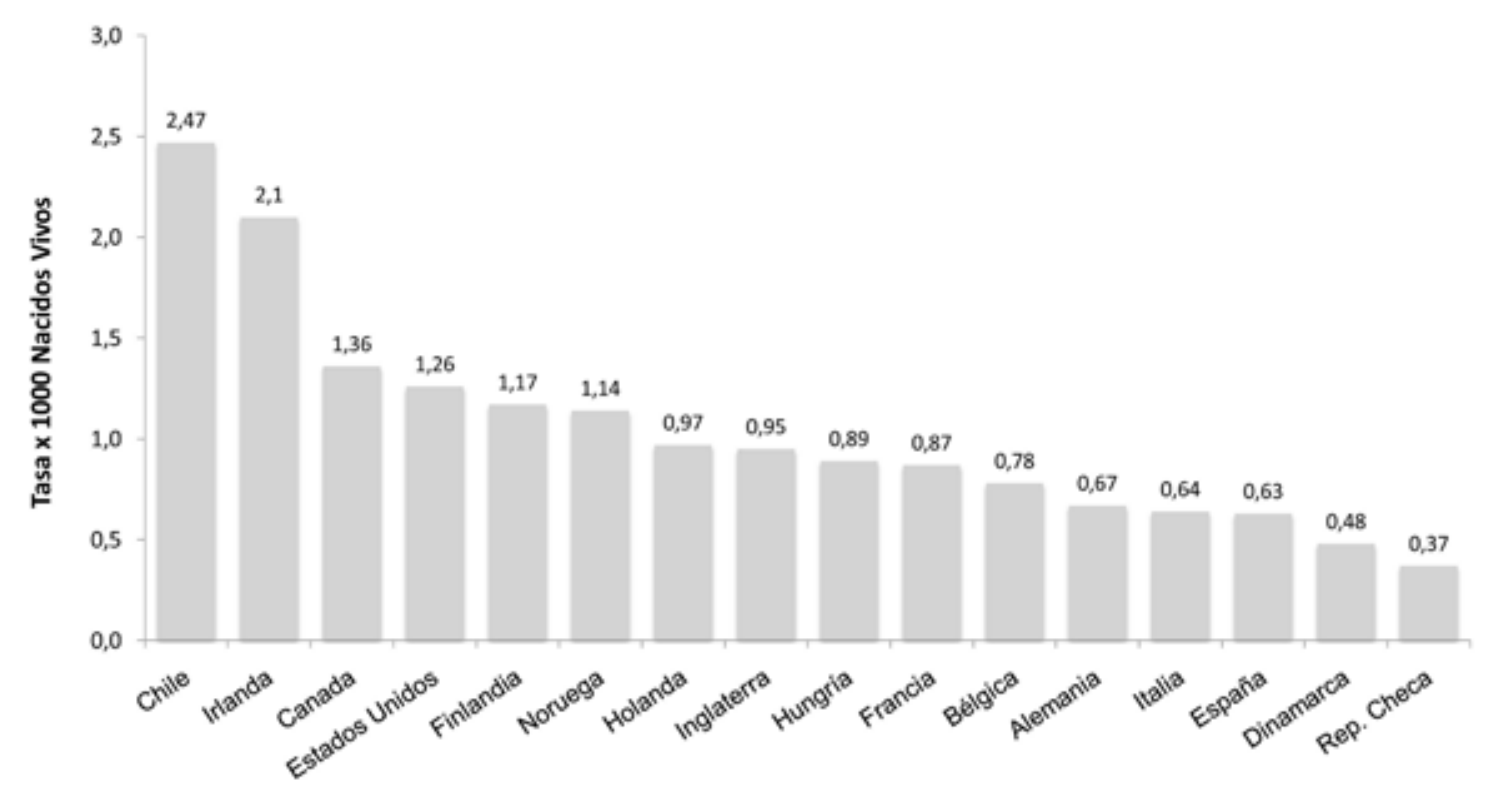

Figura 5. Tasas de nacimientos de niños con trisomía 21 (síndrome de Down) en Chile, Canadá, Estados Unidos y 13 países de Europa [29,31,34].

Otro aspecto no menos relevante en la epidemiología y prevención del aborto, es contar con estimaciones de magnitud empíricamente plausibles, especialmente para diseñar estrategias preventivas proporcionales. Un trabajo de 1990, basado en encuestas de opinión subjetivas con factores de expansión, estimó en Chile cifras tan abultadas como 160.000 abortos clandestinos por año [40]. Sin embargo, una revisión de estas metodologías mostró que poseen escasa o nula reproducibilidad y están sometidas a sesgos de selección, memoria e ideología de los sujetos entrevistados, especialmente en el cálculo de un factor multiplicador que amplifica el número de egresos por aborto observados en instituciones de salud [41]. Ejemplos en países que han modificado sus legislaciones, sugieren errores de estimación enormes. Por ejemplo, en el Distrito Federal de México, se estimaron pre-legislación más de 194.875 abortos por año; transcurridos más de 5 años post-legislación, las cifras de abortos provocados no han superado 20.000 por año [15]. En Uruguay, se estimaron pre-legislación 33.000 abortos por año [42]; luego del primer año de estadística completa post-legislación, la cifra total fue 6.676 abortos provocados [43]. Aun suponiendo algún sub-reporte en los abortos legales de estos países, estos números muestran que tales metodologías, fallan en proveer estimaciones de aborto empíricamente plausibles [44].

Aunque no se puede conocer con exactitud el número de abortos provocados en la clandestinidad, es posible realizar aproximaciones epidemio- lógicas objetivas, independientes del operador y reproducibles. La Tabla III presenta resultados de un método residual recientemente descrito [41], que estima el número de abortos provocados utilizando los nacidos vivos, probabilidades de concepción viable y aborto clínico [45,46], y egresos hospitalarios para diferentes tasas de complicaciones. Mientras mayor sea el exceso de hospitalizaciones por aborto observado y menor sea la tasa de complicaciones del método abortivo, mayor será el estimado de abortos provocados. Por ejemplo, considerando tasas de falla $30 \%$ a $40 \%$ con el uso auto-administrado de misoprostol [21-23], se estiman en promedio entre 13.553 y 18.071 abortos provocados por año, con una tasa de $16,1 \%$ de todas las hospitalizaciones por aborto en Chile (Tabla III).

Otro método más simple y accesible, es la estandarización epidemiológica indirecta, combinando tasas de poblaciones conocidas. En la Tabla IV, se han utilizado como estándar, las tasas de España para los primeros 5 años de estadística completa [41], corregidas por las diferencias de fecundidad global y edad de la población femenina chilena expuesta a riesgo para el año 2010. Se proveen intervalos de confianza de $95 \%$ para cada punto de estimación. En promedio, se estiman 18.240 abortos provocados por año con una tasa de 4,65 por cada 1000 mujeres en edad fértil. Ambos métodos llegan a resultados parecidos, descansan en información de estadísticas vitales disponibles con estimadores objetivos y evitan el uso de amplificadores de validez desconocida [44]. 


\section{Tabla III}

ESTIMACIONES DE ABORTOS ESPERADOS COMBINANDO ESTADÍSTICAS DE EGRESOS HOSPITALARIOS 2001 A 2008, NACIDOS VIVOS, PROBABILIDADES RECÍPROCAS DE CONCEPCIONES VIABLES Y ABORTOS [45,46], PARA DISTINTAS TASAS PORCENTUALES DE COMPLICACIONES. ADAPTADO DE KOCH ET AL [9]

\begin{tabular}{|c|c|c|c|c|c|c|c|c|c|}
\hline \multirow[t]{2}{*}{ Año } & \multirow{2}{*}{$\begin{array}{c}\text { Nacidos } \\
\text { vivos } \\
\text { observados } \dagger\end{array}$} & \multirow{2}{*}{$\begin{array}{c}\text { Hospitalización } \\
\text { por aborto }\end{array}$} & \multirow{2}{*}{$\begin{array}{l}\text { Abortos clínicos } \\
\text { esperados }\end{array}$} & \multirow{2}{*}{$\begin{array}{l}\text { Exceso de } \\
\text { abortos } \ddagger\end{array}$} & \multicolumn{5}{|c|}{ Abortos provocados según tasa de complicaciones $\Psi$} \\
\hline & & & & & $(\%)^{\mathrm{C}}$ & $50 \%$ & $40 \%$ & $30 \%$ & $20 \%$ \\
\hline 2001 & 248.651 & 34.479 & 29.319 & 5.160 & 15,0 & 10.321 & 12.901 & 17.201 & 25.802 \\
\hline 2002 & 241.027 & 34.968 & 28.420 & 6.548 & 18,7 & 13.097 & 16.371 & 21.828 & 32.742 \\
\hline 2003 & 236.223 & 33.497 & 27.853 & 5.644 & 16,8 & 11.288 & 14.110 & 18.813 & 28.219 \\
\hline 2004 & 232.588 & 33.835 & 27.425 & 6.410 & 18,9 & 12.821 & 16.026 & 21.368 & 32.052 \\
\hline 2005 & 232.092 & 33.184 & 27.366 & 5.818 & 17,5 & 11.636 & 14.545 & 19.393 & 29.090 \\
\hline 2006 & 233.104 & 33.145 & 27.485 & 5.660 & 17,1 & 11.319 & 14.149 & 18.865 & 28.298 \\
\hline 2007 & 242.054 & 32.532 & 28.541 & 3.991 & 12,3 & 7.983 & 9.978 & 13.304 & 19.957 \\
\hline 2008 & 248.366 & 33.423 & 29.285 & 4.138 & 12,4 & 8.276 & 10.345 & 13.794 & 20.690 \\
\hline Media & 248.366 & 33.633 & 28.212 & 5.421 & 16,1 & 10.843 & 13.553 & 18.071 & 27.106 \\
\hline
\end{tabular}

†: Basado en datos de nacidos vivos corregidos por el Instituto Nacional de Estadísticas, INE (2010) "Estadísticas Vitales, Informe anual 2008". "Datos publicados por el Ministerio de Salud de Chile. "Estimado aplicando las probabilidades de concepción viables y aborto clínico [45,46]. Las probabilidades utilizadas son de 0,67 para nacido vivo y 0,079 para aborto clínico ¥: Estimado como la diferencia entre hospitalizaciones por aborto observadas y esperadas. ${ }^{C}$ Proporción estimada de hospitalizaciones relacionada con abortos provocados. $\Psi$ : Número total absoluto de abortos provocados estimados según diferentes tasas de complicaciones desde $20 \%$ (2 de cada 10) hasta $50 \%$ (5 de cada 10) aplicadas al residuo en exceso de hospitalización por aborto.

\section{Tabla IV}

ESTIMACIÓN DE ABORTOS PROVOCADOS PARA POBLACIÓN CHILENA UTILIZANDO TASAS ESTÁNDAR DE ESPAÑA PARA LOS PRIMEROS 5 AÑOS DE ESTADÍSTICAS COMPLETAS [41]

\begin{tabular}{|c|c|c|c|c|c|c|c|c|c|}
\hline \multirow[t]{2}{*}{ Modelo $^{a}$} & \multirow{2}{*}{$\begin{array}{c}\text { Tasa } \\
\text { estándar } \\
\text { (España) }^{b}\end{array}$} & \multirow{2}{*}{$\begin{array}{l}\text { Población } \\
\text { fértil } \\
(\text { Chile })^{\mathrm{C}}\end{array}$} & \multirow{2}{*}{$\begin{array}{c}\text { Factor } \\
\text { corrección } \\
\text { Fecundidad }\end{array}$} & \multirow{2}{*}{$\begin{array}{l}\text { Factor } \\
\text { corrección } \\
\text { Edad }^{\mathrm{e}}\end{array}$} & \multirow{2}{*}{$\begin{array}{c}\text { Abortos } \\
\text { provocados } \\
\text { (crudos) }\end{array}$} & \multirow{2}{*}{$\begin{array}{c}\text { Abortos } \\
\text { provocados } \\
\text { (ajustados) }\end{array}$} & \multirow{2}{*}{$\begin{array}{c}\text { Tasa por } \\
1000 \\
\text { mujeres }\end{array}$} & \multicolumn{2}{|c|}{ IC 95\%† } \\
\hline & & & & & & & & Inferior & Superior \\
\hline 1 (1987) & 2,0193 & 3.923 .514 & 1,2199 & 0,0331 & 7.923 & 9.927 & 2,53 & 2,48 & 2,58 \\
\hline 2 (1988) & 3,1066 & 3.923 .514 & 1,2408 & 0,0297 & 12.189 & 15.486 & 3,94 & 3,88 & 4,01 \\
\hline 3 (1989) & 3,6086 & 3.923 .514 & 1,2670 & 0,0262 & 14.158 & 18.309 & 4,67 & 4,59 & 4,73 \\
\hline $4(1990)$ & 4,3547 & 3.923 .514 & 1,2880 & 0,0227 & 17.086 & 22.394 & 5,71 & 5,63 & 5,78 \\
\hline 5 (1991) & 4,8572 & 3.923 .514 & 1,3037 & 0,0195 & 19.057 & 25.216 & 6,43 & 6,34 & 6,50 \\
\hline Media & 3,6035 & 3.923 .514 & 1,2639 & 0,0262 & 14.138 & 18.240 & 4,65 & 4,581 & 4,716 \\
\hline
\end{tabular}

${ }^{a}$ Se refiere al año de la tasa oficial publicada por el INE de España utilizada como estándar. ${ }^{\text {b }}$ Tasa por 1000 mujeres en edad fértil. ${ }^{C}$ Población fértil estimada para 2010 según censo $2002 .{ }^{d}$ Calculado como recíproco de la diferencia en la tasa de fecundidad global entre Chile para el año 2010 y España entre 1987 y $1991 .{ }^{e}$ Calculado como factor proporcional de diferencia entre la población femenina en edad fértil de España y Chile. ${ }^{\dagger}$ Número absoluto de abortos luego de ajuste por fecundidad y edad. † Intervalos de confianza de $95 \%$ para la tasa de abortos provocados por 1000 mujeres en edad fértil. 
Finalmente, es necesario remarcar, que los cambios de estatus legal no están enfocados en la prevención del aborto provocado, sino solamente en facilitar su acceso dependiendo de la permisividad del tipo de legislación [6,7,9]. Tampoco resuelven el problema de vulnerabilidad específica que motiva un aborto provocado, como la coerción o el miedo. Incluso, el aborto repetido se ha transformado en un problema en varios países con legislaciones permisivas como España [44], Francia [47], Holanda [48], o Nepal [49], por ejemplo. Aún el aborto auto-provocado en la clandestinidad, como muestran estudios recientes, no es reemplazado completamente en países con aborto legal a petición, particularmente con la disponibilidad creciente de misoprostol [50].

En nuestro país, la primera línea de prevención del aborto provocado, históricamente, se ha enfocado sobre la planificación familiar mediante provisión y acceso a métodos anticonceptivos, destinada a evitar un embarazo no planificado. Cuando ésta falla o es insuficiente, una segunda línea de prevención más reciente, ha sido la emergencia de programas de apoyo para embarazos vulnerables en riesgo de aborto por parte de la sociedad civil o por equipos obstétricos locales en el caso de enfermedades congénitas graves. Expandir la cobertura de estos programas preventivos y monitorear su efectividad, es necesario para prevenir y continuar disminuyendo los abortos provocados en la clandestinidad.

Elard Koch, MPH. Epidemiólogo, Director de Investigación. Centro de Medicina Embrionaria Experimental y Salud Materna. Instituto MELISA, Concepción, Chile.

\section{REFERENCIAS}

1. Gissler M, Fronteira I, Jahn A, Karro H, Moreau C, Oliveira da Silva $M$, et al. Terminations of pregnancy in the European Union. BJOG 2012;119(3):324-32.

2. Leppälahti $S$, Gissler $M$, Mentula $M$, Heikinheimo O. Trends in teenage termination of pregnancy and its risk factors: a population-based study in Finland, 1987-2009. Hum Reprod 2012;27(9):2829-36.

3. Koch E. Impact of reproductive laws on maternal mortality: the Chilean natural experiment. Linacre $Q$ 2013;80(2):151-60.

4. Biblioteca del Congreso Nacional de Chile. Historia de la Ley No 18.826. Sustituye artículo 119 del Código Sanitario. Transcripciones y Antecedentes. [198 páginas]. Disponible en:http://www.leychile.cl/Consulta/ portada_hl?anio=1989. Acceso el 20 de agosto de 2014.

5. Donoso E. ¿Unsafe abortion en Chile? Rev Chil Obstet Ginecol 2008;73:359-61.

6. Calhoun B. The maternal mortality myth in the context of legalized abortion. Linacre Q 2013;80:264-76.
7. Thorp JM. Public health impact of legal termination of pregnancy in the US: 40 Years Later. Scientifica (Cairo) 2012;2012:1-16.

8. Viel B. Results of a family planning program in the western area of the city of Santiago. Am J Public Health Nations Health1969;59:1898-1909.

9. Koch E, Thorp J, Bravo M, Gatica S, Romero CX, Aguilera $\mathrm{H}$, et al. Women's education level, maternal health facilities, abortion legislation and maternal deaths: a natural experiment in Chile from 1957 to 2007. PLOS ONE 2012;7:e36613.

10. Koch E, Calhoun B, Aracena P, Gatica S, Bravo M. Women's education level, contraceptive use and maternal mortality estimates. Public Health 2014;128(4):384-7.

11. Valenzuela CY. [Scientific ethics of therapeutic abortion]. Rev Med Chil 2003;131(5):562-8.

12. Armijo R, Monreal T. The problem of induced abortion in Chile. Milbank Mem Fund Q1965;43:Suppl:263-80.

13. Montebruno $P$, Delgado $A$. La vía chilena hacia el aborto. Archivo The Clinic; Marzo 2012 [originalmente publicado en 2003]. Disponible en: http://www.theclinic.cl/2012/03/13/la-via-chilena/. Acceso el 20 de agosto de 2014.

14. Armijo R, Requena M. Epidemiologic aspects of abortion in Chile. Public Health Rep 1968;83(1):41-8.

15. Koch E, Aracena P, Gatica S, et al. Fundamental discrepancies in abortion estimates and abortion-related mortality: A reevaluation of recent studies in Mexico with special reference to the International Classification of Diseases. Int J Women Health 2012;4:613-23.

16. Pandya PP, Snijders RJ, Psara N, Hilbert L, Nicolaides $\mathrm{KH}$. The prevalence of non-viable pregnancy at 10-13 weeks of gestation. Ultrasound Obstet Gynecol1996;7(3):170-3.

17. Molina R, Molina T, Carrasco X, Eguiguren P. Profile of abortion in Chile, with extremely restrictive law. Open J Obstet Gynecol 2013;3:732-38.

18. Schiavon R, Troncoso E, Polo G. Analysis of maternal and abortion-related mortality in Mexico over the last two decades, 1990-2008. Int J Gynaecol Obstet 2012;118 Suppl 2:S78-86.

19. Zamberlin N, Romero M, Ramos S. Latin American women's experiences with medical abortion in settings where abortion is legally restricted. Reprod Health 2012;9(1):34.

20. Donoso E, Carvajal JA. [The change in the epidemiological profile of maternal mortality in Chile will hinder the fulfillment of the Millennium 5th goal]. Rev Med Chil 2012;140(10):1253-62.

21. Ngai SW, Tang OS, Chan YM, Ho PC. Vaginal misoprostol alone for medical abortion up to 9 weeks of gestation: efficacy and acceptability. Hum Reprod 2000;15(5):1159-62.

22. Ngoc NT, Shochet T, Raghavan S, Blum J, Nga NT, Minh NT, et al. Mifepristone and misoprostol compared with misoprostol alone for second-trimester abortion: a randomized controlled trial. Obstet Gynecol 2011;118(3):601-8.

23. Rodríguez-Cárdenas A, Velasco-Boza A. Uso de 600 $\mathrm{mg}$ de Misoprostol para inducir el aborto temprano. Rev Cubana Obstet Ginecol [online] 2003;29(1). Disponible en: http://scielo.sld.cu/scielo.php?pid=S0138$600 X 2003000100008 \&$ script=sci_arttext. Acceso el 7 de Junio de 2014. 
24. Koch E. Impact of reproductive laws on maternal mortality: recents cientific evidence from natural experiments on different populations. Lecture at the life \& family event in the United Nations, September 19th, United Nations, New York, 2013. Disponible en: http:// www.alliancedefendingfreedom.org/mdgs. Acceso el 21 de Agosto de 2014.

25. Dueñas JL, Lete I, Bermejo R, Arbat A, Pérez-Campos E, Martínez-Salmeán J, et al. Trends in the use of contraceptive methods and voluntary interruption of pregnancy in the Spanish population during 19972007. Contraception 2011;83(1):82-7.

26. Viel B, Campos W. Chilean history of infant and maternal mortality, $1940-1985$. Perspect Int Planif Fam 1987;(Spec No):24-8

27. Klick J, Neelsen S, Stratmann T. The relationship between abortion liberalization and sexual behavior: International evidence. Am Law Econ Rev (Fall 2012);14(2):457-87.

28. Koch E. Abortion prevention programs in Chile. Conference at the United Nations, September 19, New York, 2013. Disponible en: http://www.melisainstitute. com/conferences.html. Acceso el 21 de Agosto de 2014.

29. Nazer HJ, Cifuentes OL. [Congenital malformations in Latin America in the period 1995-2008]. Rev Med Chil 2011;139(1):72-8.

30. Boyd PA, Devigan C, Khoshnood B, Loane M, Garne $\mathrm{E}$, Dolk $\mathrm{H}$, et al. Survey of prenatal screening policies in Europe for structural malformations and chromosome anomalies, and their impact on detection and termination rates for neural tube defects and Down's syndrome. BJOG 2008;115(6):689-96.

31. Cocchi G, Gualdi S, Bower C, Halliday J, Jonsson B, Myrelid $A$, et al. International trends of Down syndrome 1993-2004: Births in relation to maternal age and terminations of pregnancies. Birth Defects Res A Clin Mol Teratol 2010;88(6):474-9.

32. Khoshnood B, De Vigan C, Vodovar V, Goujard J, Goffinet F. A population-based evaluation of the impact of antenatal screening forDown's syndrome in France, 1981-2000. BJOG 2004;111(5):485-90.

33. Quadrelli R, Quadrelli A, Mechoso B, Laufer M, Jaumandreu $C$, Vaglio A. Parental decisions to abort or continue a pregnancy following prenatal diagnosis of chromosomal abnormalities in a setting where termination of pregnancy is not legally available. Prenat Diagn 2007;27(3):228-32.

34. EUROCAT. European Surveillance of Congenital Anomalies. Prevalence Tables. Disponible en: http:// www.eurocat-network.eu/accessprevalencedata/prevalencetables. Acceso el 21 de Agosto de 2014.

35. Donoso E, Carvajal JA. [Eugenic abortion could explain the lower infant mortality in Cuba compared to that in Chile]. Rev Med Chil 2012;140(8):999-1005.

36. Calhoun BC, Napolitano P, Terry M, Bussey C, Hoeldtke NJ. Perinatal hospice. Comprehensive care for the family of the fetus with a lethal condition. J Reprod Med 2003;48(5):343-8.
37. Hoeldtke NJ, Calhoun BC. Perinatal hospice. Am J Obstet Gynecol 2001;185(3):525-9.

38. D'Almeida M, Hume RF, Lathorp A, Njoku A, Calhoun B. Perinatal Hospice: Family-Centered Care of the Fetus with a Lethal Condition .J Am Phys Surg 2006;11(2):52-5.

39. Neira J. Programa de cuidados paliativos perinatales UC-Christus. Primer informe de 18 meses de desarrollo. Disponible en:http://xn--acompaar-es-6db.cl/ wp/wp-content/uploads/2014/08/acompa\%C3\%B1ares-11.pdf. Acceso el 19 de Septiembre de 2014.

40. Singh S, Wulf D. Niveles estimados de aborto inducido en seis países latinoamericanos. Perspectivas Internacionales en Planificación Familiar 1994;№special: 3-13. Guttmacher Institute.

41. Koch E, Bravo M, Gatica S, Stecher JF, Aracena P, Valenzuela $S$, et al. Sobrestimación del aborto inducido en Colombia y otros países latinoamericanos. Ginecol Obstet Mex 2012;80(5):360-72.

42. Sanseviero R. Tolerancia y negación. El aborto en Uruguay. Editado por Centro Nacional de Investigación e Información para la Paz, Montevideo, 2003. Disponible en: https://www.academia.edu/1558987/ Condena_tolerancia_y_negacion._Situacion_del_ aborto_en_Uruguay. Acceso el 20 de Septiembre 2014.

43. Ministerio de Salud Pública, República Oriental del Uruguay. Interrupción voluntaria del embarazo. Diciembre 2012 - Noviembre 2013. Disponible en: http://www.msp.gub.uy/sites/default/files/archivos_ adjuntos/conferencia\%20prensa\%20IVE\%20FEBRERO\%202014.pdf. Acceso el 17 de Agosto de 2014.

44. Koch E, Aracena P, Bravo M, Gatica S, Stecher JF, Valenzuela $S$, et al. Deficiencias en la estimación de abortos para latinoamérica: Respuestas de los autores a Singh y Bankole. Ginecol Obstet Mex 2012;80(11):740-7.

45. Wilcox AJ, Weinberg CR, Baird DD. Timing of sexual intercourse in relation to ovulation. Effects on the probability of conception, survival of the pregnancy, and sex of the baby. N Engl J Med 1995;333(23):1517-21.

46. Wang X, Chen C, Wang L, Chen D, Guang W, et al. Conception, early pregnancy loss, and time to clinical pregnancy: a population-based prospective study. Fertil Steril 2003;79:577-84

47. Bajos N, Prioux F, Moreau C. Increase of repeat abortion in France: from contraceptive issues to postponement of childbearing age. Rev Epidemiol Sante Publique 2013;61(4):291-8.

48. Thapa $S$, Neupane S. Risk factors for repeat abortion in Nepal. Int J Gynaecol Obstet 2013;120(1):32-6.

49. Picavet C, Goenee M, Wijsen C. Characteristics of women who have repeat abortions in the Netherlands. Eur J Contracept Reprod Health Care 2013;18(5):32734.

50. Manouana M, Kadhel P, Koffi A, Janky E. Illegal abortion with misoprostol in Guadeloupe. J Gynecol Obstet Biol Reprod (Paris) 2013;42(2):137-42. 